\title{
Newly Admitted Psychiatric Inpatients after the 3.11 Disaster in Fukushima, Japan
}

\author{
Masaki Hisamura1, Arinobu Hori'2, Akira Wada ${ }^{3}$, Itaru Miura3 ${ }^{3}$, Hiroshi Hoshino3, \\ Shuntaro Itagaki ${ }^{3}$, Yasuto Kunii ${ }^{3}$, Junya Matsumoto ${ }^{3}$, Hirobumi Mashiko ${ }^{3}$, \\ Craig Katz ${ }^{4}$, Shin-Ichi Niwa ${ }^{5}$
}

\footnotetext{
${ }^{1}$ Department of Emergency Medicine Saitama Medical Center, Saitama Medical University, Saitama, Japan ${ }^{2}$ Department of Disaster and Comprehensive Medicine, Fukushima Medical University, Fukushima, Japan ${ }^{3}$ Department of Neuropsychiatry, Fukushima Medical University, Fukushima, Japan ${ }^{4}$ Departments of Psychiatry and Medical Education, Icahn School of Medicine at Mount Sinai, New York, USA ${ }^{5}$ Department of Psychiatry, Aizu Medical Center, Fukushima Medical University, Fukushima, Japan Email: si-niwa@fmu.ac.jp
}

How to cite this paper: Hisamura, M. Hori, A., Wada, A., Miura, I., Hoshino, H., Itagaki, S., Kunii, Y., Matsumoto, J., Mashiko, H., Katz, C. and Niwa, S.-I. (2017) Newly Admitted Psychiatric Inpatients after the 3.11 Disaster in Fukushima, Japan. Open Journal of Psychiatry, 7, 131-146. https://doi.org/10.4236/ojpsych.2017.73013

Received: April 29, 2017

Accepted: July 3, 2017

Published: July 6, 2017

Copyright (C) 2017 by authors and Scientific Research Publishing Inc. This work is licensed under the Creative Commons Attribution International License (CC BY 4.0).

http://creativecommons.org/licenses/by/4.0/

\begin{abstract}
Background: After the March 2011 "triple" disaster in Japan, the residents of Fukushima Prefecture suffered from serious psychological stress. Aims: This study aimed to elucidate the influence of stressful conditions on psychiatric disorders, as reflected in new psychiatric admissions. Methods: Diagnoses and background conditions among new psychiatric admissions during the 3 months immediately after the disaster in 2011 and the corresponding time periods of 2010 and 2012 were surveyed. Results: In 2011, more patients were admitted in confusional, manic, neurasthenic, and delirious states, whereas there were fewer admissions for depression. In 2012, more admissions pertained to depression. Twenty-four percent of the new admissions in 2011 were associated with concerns about radiation contamination and hospitalization, which declined to $4 \%$ in 2012. Conclusions: The diagnoses and background conditions among new psychiatric admissions were affected by the disaster; with the influence differing according to the time elapsed after the disaster.
\end{abstract}

\section{Keywords}

Depression, Fear of Radiation, Fukushima Disaster, Manic State, Psychiatric Inpatients

\section{Introduction}

Fukushima Prefecture sustained damage due to the earthquake and tsunami resulting from the Great East Japan Earthquake that occurred on March 11, 2011. Radioactive contamination occurred soon thereafter, due to the Fukushima 
Daiichi Nuclear Plant accident. Of the three disasters constituting the complex Fukushima disaster (i.e., the earthquake, tsunami, and nuclear accident), radioactive contamination continues to have the worst impact. The only studies on the impact of the radiation threat on psychiatric patients have been conducted on small samples by Kunii and Wada [1] [2] [3]. Therefore, we conducted a survey on trends among new psychiatric inpatients throughout Fukushima after the 3.11 complex disasters on a large sample.

\section{Method}

\subsection{Subjects}

On March 11, 2011, there were 37 facilities of either psychiatric hospitals or general hospitals with psychiatry departments in Fukushima Prefecture. However, 5 facilities were no longer able to function after the earthquake and nuclear power plant accident. Thus, among the 32 remaining facilities, we selected 30 facilities where psychiatrists of the Fukushima Society of Psychiatry worked. We requested the 30 facilities to cooperate in our planned survey on new admissions. The study sample comprised patients who had been newly hospitalized from March 12 to May 11 in 2010, 2011, or 2012. We excluded patients who had been transferred to the hospitals included in this study due to the physical damage to the hospitals they were originally admitted to or those transferred from non-medical facilities. Data from 2010 was obtained for comparison. All the administrators of the cooperating hospitals provided us their written consent to use their responses as the fundamental data in the present study.

A map indicating the hospitals under study has been shown in Figure 1. Fukushima Prefecture is divided into three regions by two mountain ranges. From east to west, the three regions are as follows: Hamadori, coastal region; Nakadori, central region; and Aizu, western region. Nakadori is further divided from north to south into northern Ken-poku, central Ken-chu, and southern Ken-nan districts. Hamadori is divided from north to south into Soso District and Iwaki District. Aizu is divided from north to south into Aizu District and Minami-Aizu District. Nine of the target hospitals were in Ken-poku, where Fukushima City is located. In April 2011, the level of environmental radiation in Fukushima City (approximately $63 \mathrm{~km}$ away from the nuclear plant) was 1.97 $\mu \mathrm{Sv} / \mathrm{h}$. Six of the target hospitals were in Ken-chu and Ken-nan districts. Koriyama City is located in Ken-chu District. The level of radiation in Koriyama City (approximately $58 \mathrm{~km}$ from the plant) was $1.79 \mu \mathrm{Sv} / \mathrm{h}$. Five of the target hospitals were in Iwaki District. The level of radiation in Iwaki City (approximately $43 \mathrm{~km}$ from the plant) was $0.36 \mu \mathrm{Sv} / \mathrm{h}$. Six of the target hospitals were in Aizu District. The level of radiation in Aizuwakamatsu City (approximately 98 $\mathrm{km}$ from the plant) was $0.16 \mu \mathrm{Sv} / \mathrm{h}$. Overall, though the levels of environmental radiation in the districts where the subjects of this study resided were low (http://www.cms.pref.fukushima.jp/download/1/7houbu0401-0430.pdf), the psychological effects among them were serious.

The status of psychiatric services in Fukushima Prefecture during the survey 


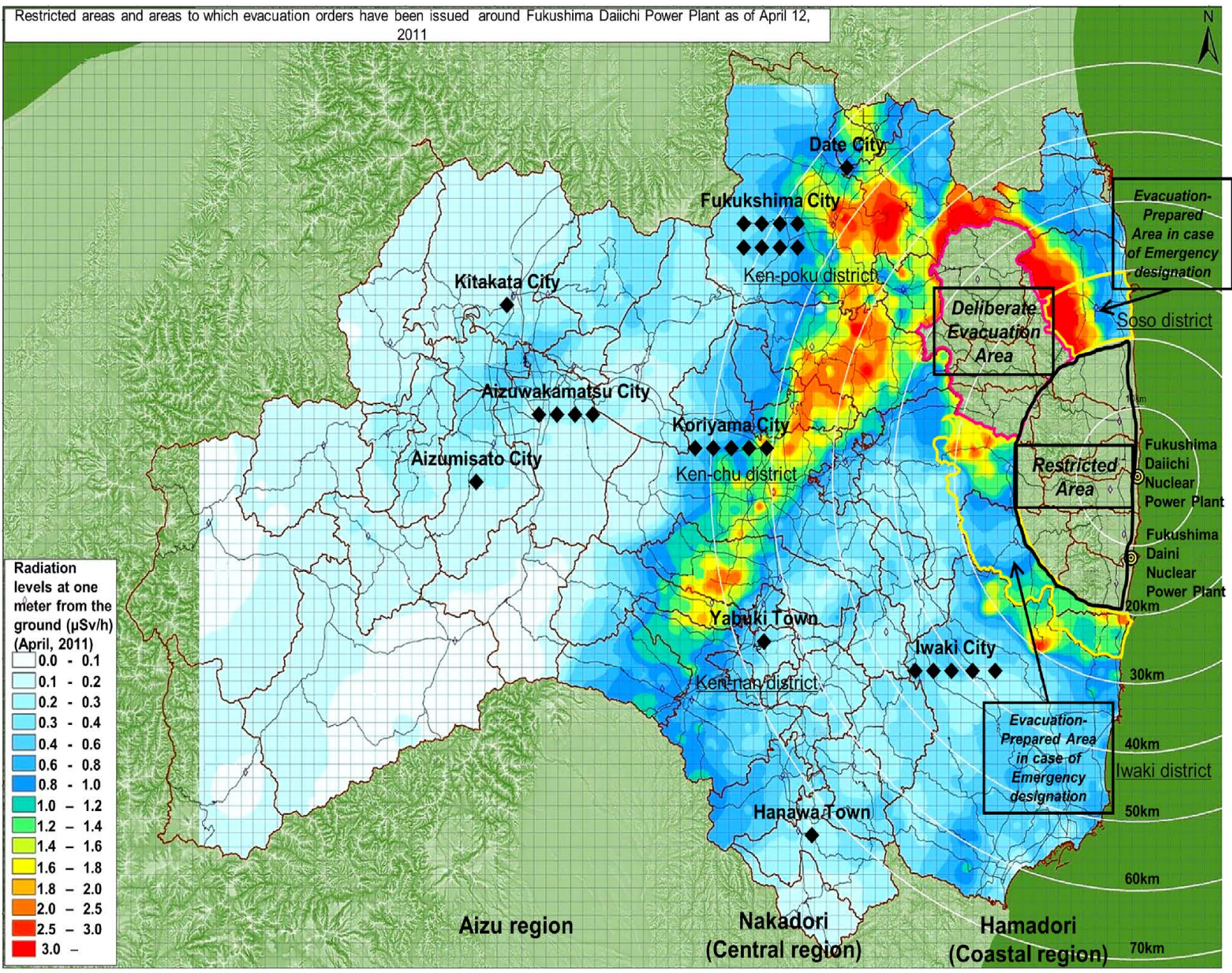

The results of monitoring of the environmental radioactivity level in the Fukushima Prefecture were expressed as microsievert/hour at $1 \mathrm{~m}$ from the ground on April 12-16, 2011 (the maps have been copied from the $5^{\text {th }}$ Fukushima Prefecture Environmental Radiation Monitoring in Mesh Survey, with the permission of Fukushima Prefecture Radiation Monitoring Unit). The italicized contents of the figure refer to the Restricted Area (surrounded by a black line), Deliberate Evacuation Area (surrounded by a red line), and the Area prepared for Evacuation in case of Emergency (surrounded by a yellow line). These areas represent the areas of the co-operating psychiatric hospitals that provided patients' data for our investigation.

Figure 1. Map of the monitored environmental radioactivity level in Fukushima Prefecture, April 12-16, 2011.

period in 2011 has been described in the paper by Kumakura [4], who reported that approximately 920 patients from 10 psychiatric hospitals had been transferred to 118 hospitals in 11 prefectures (including Fukushima Prefecture) mainly due to the designated evacuation in the Soso District. In this district, approximately 710 patients from five hospitals were evacuated, and hospitals were closed, rendering 900 hospital beds unusable [4].

\subsection{Survey Contents}

The survey examined inpatient trends immediately following the nuclear plant accident. Survey items included the number of hospitalized patients in a given district, the patients' age and gender, their diagnosis category at the time of admission, mental state at admission, type of admission, place of residence before 
admission, whether a patient's own house was damaged in the disaster, and whether fear of radiation played a role in the patient's hospitalization. The types of admission that were applicable to the patients were voluntary admission, involuntary admission, and admission instructed by the prefectural governor. The diagnosis category at admission was determined by the attending psychiatrist in accordance with the ICD-10. Mental state at admission was also determined by the attending psychiatrist by choosing from the following 11 conditions: 1) hallucinatory-paranoid state; 2) confusional state; 3) manic state; 4) depressive state; 5) neurasthenic state; 6) catatonic syndrome; 7) delirium; 8) Ganser's syndrome; 9) amnestic syndrome; 10) dementia; and 11) other. If the patient's condition fell under multiple diagnostic categories and/or mental states at admission, the option(s) that featured prominently was/were chosen. For place of residence, respondents chose one of the following options: own house, shelter, or other (including temporary house). In order to determine whether the fear of radiation exposure was one of the reasons for the hospitalization, the psychiatrist in charge interviewed and asked their patients to choose one of the following responses: association, possible association, or no association. The present study was approved by the Ethics Committee of Fukushima Medical University (No. 1434).

\subsection{Statistical Analyses}

We performed a chi-square test and a residual analysis using the Js-STAR 2012 (http://www.kisnet.or.jp/nappa/software/star/index.htm). The chi-square test was used to examine the relationship between the six factors-type of residence, ICD 10-based diagnostic category, mental state at admission, whether a patient's own house was damaged in the disaster, place of residence before admission, and level of fear regarding exposure to radiation-and the survey year $(2010,2011$, 2012). When the chi-square test indicated a significant finding, we performed a residual analysis using adjusted standardized residuals (z-scores). We deemed $z$-scores exceeding \pm 1.96 as significant, with the value of 1.96 corresponding to the $5 \%$ significance level.

\section{Results}

Among 30 institutions, we received responses from 19, 26, and 19 institutions for 2010, 2011, and 2012, respectively. After incomplete responses were excluded, results for 2010, 2011, and 2012 were obtained for 604, 610, and 606 patients, respectively. The third decimal place was rounded off and statistical results have been reported up to two decimal places.

\subsection{Summary of Newly Admitted Inpatients' Characteristics}

The mean age of the 604 patients admitted in 2010 ( 285 males and 319 females) was $54.11 \pm 21.28$ years (males: $52.39 \pm 19.44$ years, females: $55.65 \pm 22.73$ years). For 2011, the mean age for the 610 patients (299 males and 311 females) was $52.93 \pm 19.88$ years (males: $52.96 \pm 18.89$ years, females: $52.91 \pm 20.81$ 
years). For 2012, the mean age for the 606 patients ( 242 males, 364 females) was $56.52 \pm 20.32$ years (males: $56.59 \pm 19.78$ years, females: $56.47 \pm 20.70$ years).

\subsection{Newly Admitted Inpatients by District and Year}

The observed numbers and expected numbers of newly admitted patients for each district and year have been presented in Table 1. In the following results, the percentages shown before the z-scores express the value of that item as a proportion against the whole value in that year. We have used this same expression for results 4,6 , and 7 . The results of the chi-square test indicated a significant difference between the observed and expected numbers $\left[\chi^{2}(14)=284.22, p\right.$ $<0.01]$ according to new inpatients' district and the survey year (2010-2012). The chi-square test was complemented by a residual analysis. In 2011, the actual number of patients from Ken-poku District, Soso District, and Iwaki District were significantly higher than the expected number was (Ken-poku District: $26.89 \%, Z=3.46, p<0.05$; Soso District: $16.32 \%, Z=11.76, p<0.05$; Iwaki District: $14.75 \%, Z=2.53 \mathrm{p}<0.05)$. However, the actual number of patients from Ken-nan District, Aizu District, and Minami-Aizu district was significantly lower than the expected number was (Ken-nan District: $4.92 \%, \mathrm{Z}=-2.28, \mathrm{p}<0.05$;

Table 1. Newly admitted patients in eight districts in Fukushima Prefecture during 2010 to 2012 .

\begin{tabular}{cccccc}
\hline & 2010 & 2011 & 2012 & Chi-square value & p level \\
\hline Ken-poku [n (\%)] & $152(25.17)^{\mathrm{a}}$ & $164(26.89)^{\mathrm{a}}$ & $87(14.36)^{\mathrm{b}}$ & \\
Z & 2.19 & 3.46 & -5.65 & \\
Ken-chu [n (\%)] & $180(29.80)$ & $191(31.31)$ & $203(33.50)$ & \\
Z & -1.12 & -0.15 & 1.27 & \\
Ken-nan [n (\%)] & $35(5.79)$ & $30(4.92)^{\mathrm{b}}$ & $59(9.74)^{\mathrm{a}}$ & \\
Z & -1.22 & -2.28 & 3.50 & \\
Aizu [n (\%)] & $133(22.02)^{\mathrm{a}}$ & $22(3.61)^{\mathrm{b}}$ & $144(23.76)^{\mathrm{a}}$ & \\
Z & 4.54 & -10.48 & 5.97 & \\
Minami-Aizu [n (\%)] & $10(1.66)$ & $0(0.00)^{\mathrm{b}}$ & $15(2.48)^{\mathrm{a}}$ & \\
Z & 0.73 & -3.58 & 2.85 & \\
Soso [n (\%)] & $4(0.66)^{\mathrm{b}}$ & $99(16.32)^{\mathrm{a}}$ & $17(2.81)^{\mathrm{b}}$ & \\
Z & -7.19 & 11.76 & -4.60 & \\
Iwaki [n (\%)] & $67(11.09)$ & $90(14.75)^{\mathrm{a}}$ & $62(10.23)$ & \\
Z & -0.87 & 2.53 & -1.67 & \\
Outside [n (\%)] & $23(3.81)$ & $14(2.30)$ & $19(3.14)$ & \\
Z & 1.27 & -1.37 & 0.10 & \\
\hline
\end{tabular}

Z: Adjusted standardized residual; ${ }^{a}$ : The observed number is significantly larger than the expected number is (residual analysis, $\mathrm{p}<0.05$ ); ${ }^{\mathrm{b}}$ : The observed number is significantly smaller than the expected number is (residual analysis, $\mathrm{p}<0.05$ ). The observed and expected numbers of newly admitted patients for the eight districts in Fukushima Prefecture of 2010 to 2012 have been shown. The upper line for each district shows the number of admissions for that district and the parenthesized figure is the percentage expressing the proportion of total admissions accounted for by that district in that year. The lower line shows the $\mathrm{z}$-scores. 
Aizu District: 3.61\%, $Z=-10.48, \mathrm{p}<0.05$; Minami-Aizu District: 0\%, $Z=-3.58$, $\mathrm{p}<0.05)$. Similarly, in 2012, the actual number of patients from Ken-nan District, Aizu District, and Minami-Aizu District were significantly higher than the expected number was (Ken-nan District: 9.74\%, $Z=3.5, \mathrm{p}<0.05$; Aizu District: 23.74\%, $Z=5.97, \mathrm{p}<0.05$; Minami-Aizu District: 2.48\%, $\mathrm{Z}=2.85, \mathrm{p}<0.05)$. Additionally, the actual number of patients from Ken-poku District and Soso District was significantly lower than the expected number was (Ken-poku District: $14.36 \%, Z=-5.65, p<0.05$; Soso District: $2.81 \%, Z=-4.6, p<0.05$ ).

\subsection{Diagnostic Category at Admission}

Regarding the subjects' diagnostic categories (ICD-10) at admission between 2010 to 2012, with the number of subjects as units, the chi-square test indicated no difference between the observed and expected values $\left[\chi^{2}(10)=8.03\right.$, ns $]$ in terms of new inpatients' diagnostic categories at admission and the survey year.

\subsection{Mental States at Admission}

Table 2 shows the mental states of patients at admission, from 2010 to 2012,

Table 2. Subjects' mental state at admission during 2010 to 2012.

\begin{tabular}{|c|c|c|c|c|c|}
\hline & 2010 & 2011 & 2012 & $\begin{array}{l}\text { Chi-square } \\
\text { value }\end{array}$ & p level \\
\hline Hallucinatory paranoid state $[\mathrm{n}(\%)]$ & $155(25.92)$ & $146(24.01)$ & $156(25.74)$ & \multirow{16}{*}{49.31} & \multirow{16}{*}{$\mathrm{p}<0.01$} \\
\hline $\mathrm{Z}$ & 0.48 & -0.84 & 0.36 & & \\
\hline Confusional state [n (\%)] & $32(5.35)$ & $44(7.24)^{\mathrm{a}}$ & $19(3.14)^{\mathrm{b}}$ & & \\
\hline $\mathrm{Z}$ & 0.15 & 2.71 & -2.85 & & \\
\hline Manic state $[\mathrm{n}(\%)]$ & $55(9.20)$ & $71(11.68)^{\mathrm{a}}$ & $49(8.09)$ & & \\
\hline $\mathrm{Z}$ & -0.47 & 2.07 & -1.61 & & \\
\hline Depressive state $[\mathrm{n}(\%)]$ & $150(25.08)$ & $128(21.05)^{\mathrm{b}}$ & $161(26.57)$ & & \\
\hline $\mathrm{Z}$ & 0.60 & -2.24 & 1.65 & & \\
\hline Neurasthenic state $[\mathrm{n}(\%)]$ & $9(1.51)^{\mathrm{b}}$ & $39(6.41)^{\mathrm{a}}$ & $21(3.47)$ & & \\
\hline $\mathrm{Z}$ & -3.60 & 4.12 & -0.54 & & \\
\hline Delirium [n (\%)] & $17(2.84)$ & $28(4.61)^{\mathrm{a}}$ & $14(2.31)$ & & \\
\hline $\mathrm{Z}$ & -0.70 & 2.30 & -1.61 & & \\
\hline Dementia [n (\%)] & $86(14.38)$ & $71(11.68)$ & $77(12.71)$ & & \\
\hline $\mathrm{Z}$ & 1.31 & -1.12 & -0.19 & & \\
\hline Others [n (\%)] & $94(15.72)$ & $81(13.32)$ & $109(17.99)$ & & \\
\hline $\mathrm{Z}$ & 0.04 & -1.96 & 1.92 & & \\
\hline
\end{tabular}

Z: Adjusted standardized residual; ${ }^{\text {a }}$ : The observed number is significantly larger than the expected number is (residual analysis, $\mathrm{p}<0.05$ ); ${ }^{\mathrm{b}}$ : The observed number is significantly smaller than the expected number is (residual analysis, $\mathrm{p}<0.05$ ); The observed and expected numbers for the eight mental states of newly admitted patients in Fukushima Prefecture during 2010 to 2012 have been shown. The upper line for each mental state shows the number of admissions for that mental state and the parenthesized figure is the percentage expressing the proportion of total admissions accounted for by that mental state in that year. The lower line shows the $\mathrm{z}$-scores. 
with the number of subjects as units. The results of the chi-square test showed a significant difference between the observed and expected numbers according to the survey year and patients' mental state at the time of admission $\left[\chi^{2}(14)=\right.$ 49.31, $\mathrm{p}<0.01]$. The chi-square test was complemented by a residual analysis. In the period immediately following the disaster (March 12-May 11, 2011), there were significantly high numbers of patients in the confusional, manic, neurasthenic, and delirious states (confusional state: $7.24 \%, Z=2.71, \mathrm{p}<0.05$; manic state: $11.68 \%, Z=2.07, p<0.05$; neurasthenic state: $6.41 \%, Z=4.12, p<0.05$; delirious state: $4.61, Z=2.3, p<0.05$ ), and the number of depressive patients was significantly lower $(21.05 \%, Z=-2.24, \mathrm{p}<0.05)$. One year following the disaster (March 12-May 11, 2012), the number of confusional patients was significantly lower $(3.14 \%, Z=-2.85, \mathrm{p}<0.05)$. In 2012, there was an increase in the number of new patients with depression, but the difference between the observed and expected numbers did not reach a statistically significant level for the adjusted standardized residual.

\subsection{Damage to the Homes of Newly Admitted Inpatients}

Concerning the damage to new inpatients' homes at the time of admission in 2011 and 2012, the chi-square test showed no difference between the observed and expected numbers in terms of damage to their own house and the survey period $\left[\chi^{2}(1)=0.09, \mathrm{~ns}\right]$.

\subsection{Place of Residence at Admission}

Table 3(a) shows the subjects' place of residence at admission in 2011 and 2012. Regarding the residence of new inpatients before admission, we performed the chi-square test to determine whether the numbers of patients in 2011 (immediately following the disaster) and 2012 (one year following the disaster) correlated with the survey period. There was a significant difference between the observed and expected numbers in terms of patient numbers and the survey period $\left[\chi^{2}(2)=85.4, \mathrm{p}<0.01\right]$. The chi-square test was complemented by a residual analysis. In 2011, the actual number of people living in shelters was significantly higher $(13.32 \%, Z=8.63, p<0.05)$, as compared to the expected number, while the number of people living at their own house or other types of residence (e.g., group homes, nursing homes, and temporary house) was significantly lower (own house: $80.26 \%, \mathrm{Z}=-2.63, \mathrm{p}<0.05$; others: $6.41 \%, \mathrm{Z}=-4.08, \mathrm{p}<0.05$ ). In 2012 , the number of people living in their own house or in other types of residences was significantly higher than the expected number was (own house: 85.93\%, $\mathrm{Z}=2.63, \mathrm{p}<0.05$; others: $13.41 \%, \mathrm{Z}=4.08, \mathrm{p}<0.05$ ), while the number of people living in shelters was significantly lower $(0.66 \%, Z=-8.63, p<0.05)$.

\subsection{Fear of Radiation}

Table 3(b) shows whether fear of radiation was one of the reasons for the subjects' hospitalizations in 2011 and 2012. The results of the chi-square test indicated a significant difference between the observed and expected numbers in 
Table 3. (a) Subjects' residence type at admission in 2011 and 2012. (b) Fear of radiation damage as a reason for hospitalization in 2011 and 2012.

(a)

\begin{tabular}{ccccc}
\hline & 2011 & 2012 & Chi-square value & p level \\
\hline House [n (\%)] & $488(80.26)^{\mathrm{b}}$ & $519(85.93)^{\mathrm{a}}$ & & \\
Z & -2.63 & 2.63 & & \\
Shelter [n (\%)] & $81(13.32)^{\mathrm{a}}$ & $4(0.66)^{\mathrm{b}}$ & & $\mathrm{p}<0.01$ \\
Z & 8.63 & -8.63 & 85.40 & \\
Others [n (\%)] & $39(6.41)^{\mathrm{b}}$ & $81(13.41)^{\mathrm{a}}$ & & \\
Z & -4.08 & 4.08 & & \\
\hline
\end{tabular}

(b)

\begin{tabular}{ccccc}
\hline & 2011 & 2012 & Chi-square value & p level \\
\hline Association [n (\%)] & $74(12.21)^{\mathrm{a}}$ & $2(0.33)^{\mathrm{b}}$ & & \\
$\mathrm{Z}$ & 8.53 & -8.53 & & \\
Possible association [n (\%)] & $75(12.38)^{\mathrm{a}}$ & $23(3.80)^{\mathrm{b}}$ & & \\
$\mathrm{Z}$ & 5.48 & -5.48 & 110.62 & $\mathrm{p}<0.01$ \\
No association [n (\%)] & $457(75.41)^{\mathrm{b}}$ & $581(95.87)^{\mathrm{a}}$ & & \\
Z & -10.16 & 10.16 & & \\
\hline
\end{tabular}

Z: Adjusted standardized residual; a: The observed number is significantly larger than the expected number is (residual analysis, $\mathrm{p}<0.05$ ); ${ }^{\mathrm{b}}$ : The observed number is significantly smaller than the expected number is (residual analysis, $\mathrm{p}<0.05$ ); The observed and expected numbers of newly admitted patients in Fukushima Prefecture for the three residence statuses at admission, for 2010 to 2012, have been shown. The upper line for each survey item shows the number of admissions under that survey item and the parenthesized figure is the percentage expressing the proportion of total admissions accounted for by that survey item in that year. The lower line shows the z-scores.

terms of the survey year of 2011 and 2012 (i.e., one year following the disaster) $\left[\chi^{2}(2)=110.62, p<0.01\right]$. Subsequently, we performed a residual analysis to examine the adjusted residual. In 2011, the respective numbers of subjects in the "association between fear of radiation damage and hospitalization" group (association group) and the "possible association between fear of radiation exposure and hospitalization" group (possible association group) were significantly higher than the expected numbers were (association group: $12.21 \%, Z=8.53, p<0.05$; possible association group: $12.38 \%, \mathrm{Z}=5.48, \mathrm{p}<0.05)$, while the number of patients in the "no association between fear of radiation exposure and hospitalization" group (no association group) was significantly lower $(75.41 \%, Z=-10.16$, $\mathrm{p}<0.05)$. In 2012, the respective number of subjects in the association and possible association groups were significantly lower than the expected numbers were (association group: $0.33 \%, \mathrm{Z}=-8.53, \mathrm{p}<0.05$; possible association group: $3.80 \%, Z=-5.48, p<0.05)$, while the number of patients in the no association group was significantly higher $(95.87 \%, Z=10.16, p<0.05)$.

\section{Discussion}

We could obtain data from $19(59.4 \%)$ and $26(81.3 \%)$ out of the 32 psychiatric 
hospitals and general hospitals with psychiatry departments, respectively, which continued to function after the 3.11 complex disaster in Fukushima Prefecture. Therefore, we think the results of the present study represent the actual situation regarding the new admissions during the observation periods.

The observation period for each year in the present study was two months, and not all inpatients in Fukushima Prefecture were included. However, we consider the present study significant as it compares the subjects' characteristics in the pre-disaster period with those in the post-disaster period. In particular, no surveys have been conducted on psychiatric inpatients' post-disaster function, with the exception of reports by Kunii and Wada [1] [2] [3]. Therefore, the present study could provide important baseline data for follow-up surveys.

Importantly, the 2010 data on Fukushima show the same trends as that seen throughout Japan. The National Center of Neurology and Psychiatry (NCNP) affiliated to The Ministry of Health, Labor and Welfare conducted a survey on new inpatients of psychiatric hospitals in June 2010 and disclosed the proportion of the number of new inpatients by diagnostic category. According to this survey, $19.10 \%$ of them fell under the diagnostic category were F0, while $8.91 \%$, $37.73 \%, 21.97 \%$, and $5.11 \%$ belonged to the F1, F2, F3, and F4 categories, respectively (http://www.ncnp.go.jp/nimh/keikaku/630/assets/pdf/h23630.pdf).

The number of new inpatients by diagnostic category before the 3.11 disaster (for the year 2010) was the standard for comparing the changes in the number of new inpatients by diagnostic category after the 3.11 disaster (for the years 2011 and 2012). Our 2010 data almost exactly matched the 2010 national data reported by the NCNP. Thus, it is thought appropriate to use our 2010 data as a reference. In the following sections, we discuss the implications of our findings according to the major areas of focus of our survey.

\subsection{Newly Admitted Inpatients by District and Year}

In 2011, the actual number of patients from Ken-poku, Soso, and Iwaki districts was significantly higher as compared to the expected number. The increased number of newly admitted patients among people living in Ken-poku District, Soso District, and Iwaki District in 2011 likely resulted from the combined effect of highly stressful events including the earthquake, tsunami, evacuation following the nuclear disaster, and fear of radiation. Specifically, people in the Ken-poku, Soso, and Iwaki districts had potentially greater exposure of traumatic experiences than people from other areas did, which could explain the rise in psychiatric disorders and cases requiring hospitalization. Tsutsui et al. [5] conducted a survey in 2011 examining psychological stress among mothers of preschool or elementary school children. They reported that mothers from Soso, Ken-poku, and Kenchu districts had higher stress levels than mothers from other districts did. Tsutsui et al.'s study is very valuable considering the scarcity of studies that actually surveyed residents' psychological stress by district in 2011 . While the study did not report high stress levels of psychological stress in Iwaki District, the high stress levels reported in Ken-poku and Soso districts suggest 
that the above interpretation is generally correct.

On the other hand, the actual number of patients from Ken-nan, Aizu, and Minami-Aizu districts was significantly lower as compared to the expected number. Kumakura reported in 2014 that 713 Soso District patients had to be transferred to another hospital in another districts because of the earthquake disaster [6]. According to personal communication with Fukushima Prefectural Public Health and Welfare Department, 60 of the 713 patients were transferred to another psychiatric hospital in Aizu District, and 13 were transferred to a facility in Ken-nan District. The transfer of the 713 patients to facilities in Aizu and Ken-nan districts caused a decline in the number of empty beds in these districts, which reduced the availability of beds for prospective patients. Because Minami-Aizu District did not have any psychiatric hospitals prior to the disaster, many of the patients in this district who required hospitalization for psychiatric care were admitted to another psychiatric hospital in Aizu District. However, following the disaster, the psychiatric hospital beds in Aizu District were full with patients transferred from Hamadori. This reduced the opportunity for Minami-Aizu District patients to gain admission to the hospital.

\subsection{Changes in Mental States at Admission}

In the period immediately following the disaster (March 12-May 11, 2011), there were significantly higher numbers of patients in the confusional, manic, neurasthenic, and delirious states, while the number of depressive patients was significantly lower. One year following the disaster (March 12-May 11, 2012), the number of confusional patients was significantly lower, while the number of depressive patients tended to be higher.

Pre-disaster data is naturally required as a reference for comparisons to verify if there was an actual reduction in the number of new inpatients with particular psychiatric disorders after disasters. However, due to a lack of such data, most existing studies on mental health after disasters have not compared pre- and post-disaster situations. Arnberg et al. [7] used pre-disaster data on Swedish survivors of the tsunami caused by the 2004 Sumatra earthquake and found that although there was an increase in PTSD and stress-related disorders, depression had not increased. However, they did not indicate a reduction in depression after the catastrophe. Then, what factors contributed to a reduction in depression after the 3.11 complex disaster in Japan?

Haglund et al. [8] stated that six psychosocial factors protect against and aid recovery from posttraumatic stress; namely, active coping style, physical exercise, positive outlook, moral compass, social support, and cognitive flexibility. With reference to the decrease in depression after the 3.11 disaster, we believe that the highly nationalistic characteristic of Japanese people strengthened their resilience, particularly through the enhancement of Haglund et al.'s factors of "active coping style" and "social support."

At least two observations support this conclusion. First, in addition to a funeral that is conducted at the time of victim's death, the neighbors and relatives 
of the deceased regularly share their sorrow with the bereaved family, and the bereaved family is supported by the society; thus strengthening social support. Second, as reflected in the motto of "promoting bonding for mutual support" being prominently posted on social media, Japanese society came together to collectively overcome the ravages of the disaster, reflecting both active coping and social support. We believe that the prevalence of active coping and social support built resilience, resulting in the reduction of the incidence of depression. However, it is unclear why the strengthening of resilience had a stronger effect on depression than on other psychiatric conditions.

Regarding reactions of psychiatric patients to a nuclear accident, Bromet et al. [9] reported their survey results in the case of the Three Mile Island (TMI) accident. They found no significant differences between the TMI group and the comparison group. While, the increase in the number of patients with mania following the Fukushima disaster has been reported [1] [2] [3]. In addition, the exacerbation of symptoms in patients with bipolar disorder due to a catastrophic disaster has also been reported previously [10]. An increase in the number of patients in the confusional state due to the stress associated with the disaster was observed in 2011. In 2012, the chaos/confusion that emerged in the wake of the disaster appeared to be abating. Many residents left their evacuation shelters and moved into their own house or into temporary housing, resulting in a decline in environmentally derived psychological stress, and thus, a decline in overall stress [11]. The state of confusion would have declined accordingly.

Disaster-derived stress had a particularly destructive impact on the elderly. When the disaster disrupted or destroyed the environment of the elderly, they found it difficult to cope with the changes, resulting in a rise in stress and some cases of delirium [12]. Our results indicated a rise in delirium following the disaster that is consistent with the existing study. The various types of disaster-derived stress gave rise to extreme fatigue and exhaustion in many cases. The number of people who experienced neurasthenic symptoms (e.g., muscle pain, muscle contraction-derived headaches, dizziness and sleep-disorder) increased and this rise could explain the increase in hospital admissions. A study of the Chernobyl nuclear disaster by Loganovsky et al. [13] in which structured interviews were used, showed an increase in the number of depressive individuals even 18 years following the accident. Our study detected a rise in depression in 2012 , one year after the disaster, possibly reflecting the start of a similar longterm trend.

\subsection{Damage to the Homes of Newly Admitted}

Most of the residents living in evacuation centers or temporary housing were evacuees from Soso District. Living in evacuation centers creates a great deal of psychological stress [14]. This could explain the large number of new admissions from evacuation centers in 2011. According to the Reconstruction Agency data, the 6105 evacuees in evacuation centers on June 2, 2011 decreased to zero on March 8, 2012 (http://www.reconstruction.go.jp/topics/120413hinansya.pdf), 
which explains the marked decline in the number of new admissions from evacuation centers in 2012.

In 2012, an increasing proportion of evacuees had started living in temporary housing or rented accommodations. Many of them felt uneasy and isolated because of living in unfamiliar locations and circumstances. They also felt a sense of loss, as they were no longer able to return to their original homes because of the nuclear accident. Factors such as uneasiness, loneliness, and a sense of loss led to higher stress levels than before the disaster. This may explain the increase in admissions among people from other types of residence, including temporary housing. It is worth citing Nishizawa et al. [11] as an example of studies which objectively demonstrated that living in temporary housing is highly stressful. Nishizawa et al. measured blood pressure in hypertensive patients to determine how blood pressure fluctuates depending on the living environment following the 3.11 earthquake. They found that patients living in temporary housing had higher systolic blood pressure compared to those living in their own home, and they attributed this difference to living environment. Stress arising from the living environment may contribute to the increase in admissions in 2012 among those living in temporary housing.

\subsection{Fear of Radiation}

The International Commission on Radiological Protection (ICRP) recommended that radiation exposure during normal periods should be limited to no more than $1 \mathrm{mSv} /$ year, and radiation exposure due to accidents in states of emergency should not exceed $20-100 \mathrm{mSv} / \mathrm{year}$, and in the restoration period following resolution of the accident, radiation exposure should not exceed $20 \mathrm{mSv} /$ year [15]. According to the preliminary results of the Basic Survey (radiation dose estimates) of Fukushima Health Management Survey, Radiation Medical Science Center, Fukushima Medical University, the estimated radiation dose of external exposure (integrated dose for the 4 months immediate period after the accident) among $99.3 \%$ of 14,412 residents in Yamakiya District (Kawamata Town), Namie Town, and Lidate Town, who participated in the survey, was less than $10 \mathrm{mSv}$, with the maximum value in the remaining $0.7 \%$ residents being 25.1 $\mathrm{mSv}$. Previous epidemiological surveys have not confirmed any clear negative health effects of radiation exposure of less than $100 \mathrm{mSv}$. Since these were aggregate values in areas considered to contain relatively higher levels of environmental radiation, the levels of external radiation exposure for the vast majority of residents of other areas of Fukushima Prefecture is considered to be lower (Fukushima Prefecture homepage:

http://www.pref.fukushima.jp/imu/kenkoukanri/250820siryou1.pdf). Thus, conveying that radiation exposure from 3.11 is not expected to result in any clear damage to health in many areas of Fukushima Prefecture (aside from a part of the evacuation area) is considered an extremely important method for dispelling unrest. However, as shown in Table 3(b), many of the admissions in 2011 were related to fear of radiation damage. This phenomenon probably occurred from a 
deficit in the communication about the risk of radiation.

Since the Fukushima disaster, a systematized support system has been developed, based chiefly around Fukushima Medical University and the Institute for Traumatic Stress. This support system is aimed at countering excessive fears of radiation damage through means such as disseminating accurate information about the effects of radiation and studying internal radiation exposure using whole-body counters [16]. Conveying accurate information regarding radiation damage reduces negative perceptions [17]. Possibly as a result of these outreach activities, one year after 3.11 there was a sharp decrease in hospitalizations that were associated with a fear of radiation damage.

However, the fear of radiation damage originates from radiophobia, and is perceived in relation to the atomic bombings of Hiroshima and Nagasaki. In addition, studies conducted in the aftermath of the nuclear accident in Chernobyl have shown that the various mental symptoms that stem from anxiety in relation to radiation damage last over a long period of time [18]. After the Three Mile Island (TMI) nuclear accident, Bromet et al. [9] considering TMI as dangerous was significantly associated with strong distress among psychiatric patients in the TMI area. These findings suggest that it is necessary to examine physical and psychological measures for dealing with radiation exposure. Additionally, it is necessary to continuously convey accurate information regarding the risks associated with exposure to 3.11 radiation and future studies should examine the longitudinal trajectory of 3.11 radiation fears and inform governmental efforts to address these fears.

\subsection{Methodological Limitation}

There are some methodological limitations in the present study. Firstly, we did not have data for the years prior to 2010. Therefore, we compared 2011 and 2012 data with that of 2010, assuming that it represented an average year. This assumption may create bias. Another methodological limitation is related the number of hospitals participating in the present study. We asked 30 identical hospitals to cooperate in the survey across 2010, 2011, and 2012. And 19, 26, and 19 hospitals cooperated in the survey for the respective years. The nineteen hospitals included in the 2010 and 2012 data were the same, and they were included in the 26 participating hospitals for 2011. If we could have obtained data from the same 26 hospitals of across all three years, our findings may have been more generalizable.

\section{Acknowledgements}

This report is a reflection of our clinical activities before, during, and after the complex Fukushima disaster, and therefore, could have been written by any of our colleagues. Thus, the authors wish to express their sincere gratitude to all of their colleagues whose devotion, professionalism, and dedication were essential in the conditions that resembled a battlefield in the ward, but who are not listed as the authors of this manuscript. This study was supported by the SENSHIN 
Medical Research Foundation.

We wish to express our sincere thanks to the hospitals which cooperated with us in the present study for providing us the fundamental data on new admissions. The alphabetical order list of the psychiatric hospitals where the psychiatrists belonging to the "Fukushima Prefecture Psychiatric Care Survey Group" worked has been presented below. In the parentheses following hospital names, their location areas in Fukushima Prefecture have been indicated.

Aizunishi Hospital (Aizu District, Aizu)

Aratame Hospital (Iwaki District, Hamadori)

Asaka Hospital (Ken-chu District, Nakadori)

Fuji Hospital (Ken-poku District, Nakadori)

Fuksuhima Matsugaoka Hospital (Ken-poku District, Nakadori)

Fukushima Medical University Hospital (Ken-poku District, Nakadori)

Fukushima Prefectural Aizu Genral Hospital (Aizu District, Aizu)

Fukushima Prefectural Yabuki Hospital (Ken-nan District, Nakadori)

Fukushima Red Cross Hospital (Ken-poku Disrict, Nakadori)

Hanawa Kousei Hospital (Ken-nan Dsitrict, Nakadori)

Haryugaoka Hospital (Ken-chu District, Nakadori)

Hoshigaoka Hospital (Ken-chu District, Nakadori)

Ichiyokai Hospital (Ken-poku District, Nakadori)

IIzuka Hospital (Aizu District, Aizu)

Itakura Hospital (Ken-poku District, Nakadori)

Iwaki Kaisei Hospital (Iwaki District, Hamadori)

Izumi Hoyoin, Hamadori (Iwaki District, Hamadori)

Jusendo-Shonan Hospital (Ken-chu District, Nakadori)

Maikohama Hospital (Iwaki District, Hamadori)

Murakami Hospital (Ken-poku District, Nakadori)

Nagahashi Hospital (Iwaki District, Hamadori)

Ohta General Hospital (Ken-chu District, Nakadori)

Sakuragaoka Hospital (Ken-poku District, Nakadori)

Shimizu Hospital (Ken-poku District, Nakadori)

Takada Kousei Hospital (Aizu District, Aizu)

Takeda General Hospital (Aizu District, Aizu)

Tsuruga Matsukubo Hospital (Aizu District, Aizu)

\section{References}

[1] Kunii, Y., Wada, A., Matsumoto, J., Yabe, H. and Niwa, S. (2012) Worsening of Manic State in Patients with Bipolar I Disorder Following the Fukushima Disaster. Psychiatry and Clinical Neurosciences, 66, 622-623. https://doi.org/10.1111/j.1440-1819.2012.02409.x

[2] Wada, A., Kunii, Y., Matsumoto, J., Itagaki, S., Yabe, H., Mashiko, H. and Niwa, S. (2013) Changes in the Condition of Psychiatric Inpatients after the Fukushima Disaster. Fukushima Journal of Medical Science, 59, 39-42.

[3] Matsumoto, J., Kunii, Y., Wada, A., Mashiko, H., Yabe, H. and Niwa, S. (2014) Mental Disorders that Exacerbated due to the Fukushima Disaster: A Complex Ra- 
dioactive Contamination Disaster. Psychiatry and Clinical Neurosciences, 68, 182187. https://doi.org/10.1111/pcn.12112

[4] Kumakura, T. (2013) Fukushima-ken seishinka byoin kyokai kaiin byoin no hisai/higai to fukkyu.fukko (Damage to and Reconstruction of Fukushima Psychiatric Hospitals Association member hospitals). Higashi Nihon Daishinsai Kirokushu (Great East Japan Earthquake Compilation). Fukushima Medical Association, Fukushima, 266-269. (in Japanese)

[5] Tsutsui, Y. (2015) Psychological Problems Caused by Fukushima Dai-Ichi Nuclear Plant Accident. Advances in the medicine on developmental disabilities. Progress in Medicine for Developmental Disorders, 27, 33-44. (in Japanese)

[6] Kumakura, T. (2014) The Actuality and Challenges for the Future of the Great East Japan Earthquake and Nuclear Plant Accident in Fukushima Prefecture. Journal of Japanese Association of Psychiatric Hospitals, 33, 16-22. (in Japanese)

[7] Arnberg, F.K., Gudmundsdóttir, R., Butwicka, A., Fang, F., Lichtenstein, P., Hultman, C.M. and Valdimarsdóttir, U.A. (2015) Psychiatric Disorders and Suicide Attempts in Swedish Survivors of the 2004 Southeast Asia Tsunami: A 5 year Matched Cohort Study. Lancet Psychiatry, 2, 817-824. https://doi.org/10.1016/S2215-0366(15)00124-8

[8] Haglund, M., Cooper, N., Southwick, S. and Charney, D. (2013) 6 Keys to Resilience for PTSD and Everyday Stress. Current Psychiatry, 6, 23-30.

[9] Bromet, E., Schulberg, H.C. and Dunn, L. (1982) Reactions of Psychiatric Patients to the Three Mile Island Nuclear Accident. Archives of General Psychiatry, 39, 725730. https://doi.org/10.1001/archpsyc.1982.04290060069014

[10] Aronson, T.A. and Shukla, S. (1987) Life Events and Relapse in Bipolar Disorder: The Impact of a Catastrophic Event. Acta Psychiatrica Scandinavica, 75, 571-576. https://doi.org/10.1111/j.1600-0447.1987.tb02837.x

[11] Nishizawa, M., Hoshide, S., Okawara, Y., Shimpo, M., Matsuo, T. and Kario, K. (2015) Aftershock Triggers Augmented Pressor Effects in Survivors: Follow-Up of the Great East Japan Earthquake. American Journal of Hypertension, 28, 14051408. https://doi.org/10.1093/ajh/hpv044

[12] Cacchione, P.Z., Culp, K., Laing, J. and Tripp-Reimer, T. (2003) Clinical Profile of Acute Confusion in the Long-Term Care Setting. Clinical Nursing Research, 12, 145-158. https://doi.org/10.1177/1054773803012002003

[13] Longanovsky, K., Havenaar, J.M., Tintle, N.L., Guey, L.T., Kotov, R. and Bromet, E.J. (2008) The Mental Health of Clean-Up Workers 18 Years after the Chernobyl Accident. Psychological Medicine, 38, 481-488.

[14] Suzuki, Y., Fukasawa, M., Obara, A. and Kim, Y.J. (2014) Mental Health Distress and Related Factors among Prefectural Public Servants Seven Months after the Great East Japan Earthquake. Epidemiology, 24, 287-294.

https://doi.org/10.2188/jea.je20130138

[15] International Commission on Radiological Protection (2011) Fukushima Nuclear Power Plant Accident.

[16] Yasumura, S., Hosoya, M., Yamashita, S., Kamiya, K., Abe, M., Akashi, M., Kodama, K., Ozasa, K. and Fukushima Health Management Survey Group (2012) Study Protocol for the Fukushima Health Management Survey. Journal of Epidemiology, 22, 375-383. https://doi.org/10.2188/jea.JE20120105

[17] Rubin, G.J., Amlôt, R., Wessely, S. and Greenberg, N. (2012) Anxiety, Distress and Anger among British Nationals in Japan Following the Fukushima Nuclear Accident. British Journal of Psychiatry, 201, 400-407. 
https://doi.org/10.1192/bjp.bp.112.111575

[18] Shigematzu, I. (1991) The International Chernobyl Project: An Overview. Assessment of Radiological Consequences and Evaluation of Protective Measures. International Advisory Committee, International Atomic Energy Agency, Vienna.

Submit or recommend next manuscript to SCIRP and we will provide best service for you:

Accepting pre-submission inquiries through Email, Facebook, LinkedIn, Twitter, etc. A wide selection of journals (inclusive of 9 subjects, more than 200 journals)

Providing 24-hour high-quality service

User-friendly online submission system

Fair and swift peer-review system

Efficient typesetting and proofreading procedure

Display of the result of downloads and visits, as well as the number of cited articles

Maximum dissemination of your research work

Submit your manuscript at: http://papersubmission.scirp.org/

Or contact ojpsych@scirp.org 